\title{
Editorial
}

\section{Advancing safe and healthy work for all ages}

The year 2020 marks the first time in human history when the number of adults $60 \mathrm{yr}$ and older is projected to exceed the number of children younger than five years of age $^{1)}$. This remarkable change in global age structure will continue into the foreseeable future and has profound implications for the many countries with a declining birth rate and an adult population that is growing older. At the heart of the matter is the need for longer and healthier working lives. This editorial provides a brief overview of the aging workforce issue and describes current efforts at NIOSH in the United States focusing on the goal of productive aging.

One general strategy, adopted by a growing number of countries, is to adjust social and economic policies (e.g., retirement age, pensions) to create incentives for working longer. A complementary, but more organization-based approach, is to adapt the workplace and the nature of work itself to maintain and enhance the safety, health, wellbeing, and productivity of workers as they age. Both strategies will undoubtedly play important roles in the future and will be informed by the growing knowledge base on productive aging and work.

Research from both the lab and workplace indicates that the effects of aging are often gradual, variable, and com$\mathrm{plex}^{2)}$. In many workplaces, older workers are valued for their expertise and problem-solving ability, organizational loyalty, attentiveness when it comes to safety matters, and higher levels of job satisfaction. At the same time, aging is accompanied by increased vulnerability to environmental hazards, reflected in higher rates of fatal/severe injuries in many industries, increased risk of some non-fatal injuries (e.g., slips, trips, and falls), and higher prevalence of chronic health conditions (e.g., arthritis, diabetes) and disability. This paradox of gains and losses poses both opportunities and challenges as the workforce continues to age.

In September of 2015, NIOSH established the National Center for Productive Aging and Work (NCPAW) within its Office for Total Worker Health ${ }^{\circledR}$. Although the United States is slightly younger than several other developed countries (in recent years, the expected increase in U.S. life expectancy has actually stalled ${ }^{3)}$ ), the accumulating pressures produced by shrinking birth rates and longer lifespans over many years are similar. The concept of productive aging is based on the work of Robert N. Butler, founding Director of the National Institute on Aging, and represents a positive response to an earlier, but widespread, view of aging as an inevitable process of decline and of growing isolation from society. In light of the longevity revolution, Butler and others argued that society can no longer afford to ignore the important contributions, both social and economic, that older adults can continue to make ${ }^{4}$. In order to age productively, a basic level of health and functioning is necessary, as well as an environment that promotes engagement and the opportunity to contribute.

The NCPAW approach to productive aging has four principal elements ${ }^{5)}$ : a life-span perspective that views aging as a continuous and dynamic process across the entire working life, a comprehensive, holistic approach to occupational health, consistent with its roots in Total Worker Health, that integrates factors across multiple domains (e.g., individual, physical work environment, organization), an emphasis on mutually beneficial outcomes that addresses the needs of workers (e.g., meaningful, secure work) and employers (e.g., reduced health-related costs), and $\boldsymbol{a}$ concern for multi-generational issues (e.g., mentoring, agediverse teams) that leverage the strengths of an inclusive organizational culture. As in Butler's original formulation, the term productive is interpreted broadly and includes contributions to one's own development and growth, to the employer or organization, to one's co-workers, and to society overall. Workers may encounter greater health risks with age, but given supportive working conditions and culture, they can remain healthy and valuable members of their organizations.

Current NCPAW activities include establishing research priorities for NIOSH regarding aging, working with external partners to examine aging-related issues impacting specific occupational groups (e.g., healthcare, manufacturing), and developing communication products that accurately reflect current knowledge on aging, and translate that knowledge into practical workplace strategies for advancing productive aging. The last activity is especially important given the growing scientific literature on aging and work, and the need to dispel common misconceptions that seem to persist in some organizations (e.g., older workers are unable to learn new skills).

For NCPAW and NIOSH, the range of workplace topics relevant to productive aging is broad, but there are at least three principal categories that stand out:

- Best Practices and Sustainable Interventions: Although many organizations are aware of the issues posed by an aging workforce, relatively few are taking proactive steps to address these issues ${ }^{6}$. Effective workplace 
programs need not be expensive or complicated ${ }^{7)}$. Case studies describing and, in some cases, evaluating agefriendly practices are becoming increasingly common ${ }^{8)}$, although more examples of both successes and failures across different industries are needed. Equally important are well-designed intervention studies that systematically assess short- and long-term impact and examine contextual factors that are necessary for success.

- Occupational Health Disparities: Aging workers are not a uniform population and variability in functioning and health tends to increase over the lifespan. Certain subgroups of workers or workers within some occupations may be especially vulnerable to adverse safety, health, and well-being outcomes. Aging workers who are in physically demanding jobs, who feel forced to remain in the workplace due to financial or health care needs (i.e., job $l_{\text {ock }}{ }^{9)}$ ), or those who are in low skill, low wage jobs may be especially at risk. Gender, ethnicity, and other demographic variables can also affect the trajectory of age-related changes at work. More research is needed on understudied or at risk groups with the goal of customizing programs and policies so they better meet the needs of an increasingly diverse aging workforce.

- Changing Nature of Work: As the workforce ages, the nature of work in many countries will also be experiencing a rapid transformation. Sometimes referred to as the fourth industrial revolution ${ }^{10)}$, a driving factor will be the accelerating growth of emerging technologies such as robotics, artificial intelligence, virtual reality, 3-D printing, autonomous driving, and nanotechnology. The impact of these technologies will vary by country and industry but is likely to: reduce the need for human workers in some occupations, require the learning of new skills, encourage new work arrangements, and involve exposure to a mosaic of old and new occupational hazards. Whether aging workers are left behind (e.g., replaced by automation) or utilized for their unique capabilities will depend on how these technological advances are planned and implemented. By better understanding the full range of consequences, organizations and societies can help shape the future of work so that it is as positive and inclusive as possible.

The World Health Organization (WHO) has designated 2020-2030 as the Decade of Healthy Ageing ${ }^{11)}$. Given the central role that work plays in many societies, the workplace has the potential to make a significant, if not pivotal, contribution towards achieving that goal. The challenges and opportunities posed by an aging workforce are truly global in scope and there is much that we can learn from one another regarding the advancement of sustainable work and healthier working lives. By renewing our focus on the importance of how work is designed and organized and the environment - both physical and psychosocialin which it takes place, a commitment to productive aging can benefit workers of all ages.

\section{Disclaimer}

The findings and conclusions in this editorial are those of the author and do not necessarily represent the official position of the National Institute for Occupational Safety and Health, Centers for Disease Control and Prevention.

\section{References}

1) United Nations, Department of Economic and Social Affairs, Population Division (2019). World Population Prospects 2019, custom data acquired via website.

2) Grosch JW, Hecker S, Scott K, Scholl JC (2019). Productive aging and work. In: Total Worker Health: Integrated prevention strategies in occupational safety and health, HL Hudson, JAS Nigam, SL Sauter, LC Chosewood, AL Schill, John Howard (Eds.) 229-246, American Psychological Association, Washington DC.

3) Woolf SH, Schoomaker H (2019) Life expectancy and mortality rates in the United States, 1959-2017. JAMA 322, 1996-2016. [Medline] [CrossRef]

4) Butler RN, Gleason HP (Eds.) (1985) Productive aging: Enhancing vitality in later life. Springer, New York.

5) Schulte PA, Grosch J, Scholl JC, Tamers SL (2018) Framework for considering productive aging and work. J Occup Environ Med 60, 440-8. [Medline] [CrossRef]

6) SHRM (2015). Preparing for an aging workforce. Society for Human Resource Management, Alexandria.

7) European Agency for Safety and Health at Work (2017) Healthy workplaces for all ages: Healthy workplaces good practice awards 2016-2017. Publications Office of the Euron, Luxembourg. https://osha.europa.eu/en/toolsand-publications/publications/healthy-workplaces-goodpractice-awards-2016-2017-booklet.

8) Loch CH, Sting FJ, Bauer N, Mauermann H (2010) How BMW is defusing the demographic time bomb. Harv Bus Rev 88, 99-102. [Medline]

9) Fisher GG, Ryan LH, Sonnega A, Naudé MN (2016) Job lock, work, and psychological well-being in the United States. Work Aging Retire 2, 345-58. [Medline] [CrossRef]

10) Schwab K (2016). The fourth industrial revolution. World Economic Forum.

11) World Health Organization (2019). Decade of healthy ageing 2020-2030. https://www.who.int/docs/defaultsource/decade-of-healthy-ageing/full-decade-proposal/ decade-proposal-fulldraft-en.pdf?sfvrsn=8ad3385d_6.

\section{James W. GROSCH}

Co-Director, National Center for Productive Aging and Work National Institute for Occupational Safety and Health Centers for Disease Control and Prevention USA 